\title{
474589 - EPIDURALS FOR PROSTATECTOMY DO NOT REDUCE PSA RECURRENCE
}

\author{
Sunita Broemling, MD, Ban Tsui, MD, MSc, FRCPC \\ Anesthesiology \& Pain Medicine, University of Alberta, Edmonton, AB, Canada
}

Introduction: Immunosuppression may contribute to cancer recurrence after curative surgery.(1) The immunosuppression is likely multi-factorial and may be affected by blood transfusions and overall surgical stress. Regional anesthesia may reduce the surgical stress response and positively modulate immunity. Using paravertebral blocks has recently shown to decrease the risk of recurrence or metastasis following breast cancer surgery.(2) We have previously shown that hypotensive epidural anesthesia (targeted mean arterial pressure 55-60 mmHg) can reduce blood loss and transfusions in patients undergoing radical prostatectomy.(3) The objective of this study was to retrospectively evaluate whether the incidence of biochemical recurrence of prostatespecific antigen (PSA) was lower at 5-year follow-up in those radical prostatectomy patients who, previously enrolled in our epidural study, received the epidural/general anesthesia hypotensive regime, as compared to general anesthesia alone.

Methods: Ethics approval was obtained to review the medical charts of 99 patients who previously (5 years or more), during participation in our epidural study, underwent curative radical prostatectomy due to prostate adenocarcinoma under either general anesthesia (control group; $n=50$ ) or combined epidural/general anesthesia (study group; $\mathrm{n}=49$ ). No patient received preoperative hormonal or radiation therapy. The primary outcome was the biochemical recurrence of serum PSA at any time point, using a posttreatment PSA value $>0.2 \mathrm{ng} / \mathrm{ml}$ shown to predict clinical recurrence (4). The secondary outcome was the rate of PSA recurrence as associated with low or high risk pretreatment prognosis: histological Gleason score $\leq 6$, PSA level $<10 \mathrm{ng} / \mathrm{ml}$, and tumor stage $<$ T3 were considered low risk, and any patient with a higher value in any category was placed in the high risk group. Patients who received either hormonal therapy or salvage radiotherapy since the surgery were excluded. Data are expressed as proportions and compared with Chi-square analysis.

Results: Of the 99 patients, 9 were lost to follow up with 47 and 43 patients remaining in the control and study groups respectively. The groups had similar patient demographics, preoperative PSA values, pathological tumor staging and numbers of low versus high risk patients (9 vs 12 low and 38 vs 31 high risk in control and study groups, respectively). The rate of PSA recurrence $>0.2 \mathrm{ng} / \mathrm{ml}$ was not significantly different between control and study groups ( $38 \%$ vs $40 \% ; \mathrm{P}=0.846$ ), or for patients in low (33\% vs $25 \%$; $\mathrm{P}=0.690$ ) versus high ( $39 \%$ vs $45 \% ; \mathrm{P}=0.600$ ) risk categories in either group. Follow up time was $69 \pm 23$ months.

Discussion: Long-term follow up of radical prostatectomy patients failed to demonstrate a similar benefit of regional anesthesia on cancer recurrence as for those patients with breast cancer.

References: 1) Curr Opin Anaesthesiol 2001;14:685-91, 2) Anesthesiology 2006;105:660-4, 3) Can J Anaesth 2006;53:873-80, 4) Urology 2003;61:365-9 